\title{
ON APPROACH OF MULTIPLE FREQUENCY ACTIVE VIBRATION CONTROL UNDER THE ACTUATOR WITH MAGNETIC HYSTERESIS LOOP
}

\author{
Chao Li Jianqin Mao
}

\author{
The Seventh Research Division \\ Beijing University of Aeronautics \& Astronautics, 100083, Beijing, China. \\ E-mail:lichao7715@163.com,dumao@public.bta.net.cn
}

\begin{abstract}
Since the magnetostrictive actuator is a nonlinear plant with magnetic hysteresis loop, its multiple frequency active vibration control is more complicated. In this paper, a Simple Hyperstable Adaptive Recursive Filter (SHARF) algorithm based on nonlinear model is proposed. The nonlinear model of magnetostrictive actuator is obtained by using radial basis neural network. The experimental result show that the proposed approach is real-time efficient. An attenuation of about $30 \mathrm{~dB}$ is achieved. The algorithm is also suitable as a reference to the stabilizing control of a class of nonlinear system with magnetic hysteresis loop. Copyright (C) 2005 IFAC
\end{abstract}

Keywords: magnetostrictive actuator, active vibration control, SHARF, radial basis neural network, magnetic hysteresis loop.

\section{INTRODUCTION ${ }^{1}$}

Micro-displacement and micro-vibration control have been investigated for years. The magnetostrictive materials have been used to reduce the vibration of structure by active control techniques.

Because of the nonlinear property of this material, several control algorithms were discussed in active vibration control, including the neural network (Michael 1993), adaptive acceleration feedback control (Z. Jason Geng 1995) and P-operator (X.Tan 2001).

Also other techniques of signal process have been investigated for active vibration control, including

\footnotetext{
${ }^{1}$ The research is support by the Basic Pre-Research Project for National Defense of P. R. China (K1200060301), the National Natural Science Foundation of China (NSFC) (90205012 and 10276005), and the National-Key Project for Basic Research of P. R. China (G2002cb312205-04).
}

adaptive $\quad \mathrm{x}$-Filter Least-Mean-Squares (x-LMS) algorithm (Z. J Geng 1994) and some kind of Infinite Impulse Response (IIR) Filters algorithm.

Landau introduced an unbiased output error procedure for seeking parameter estimates of an ARMA plant, the hyperstable output error identifier. From this, a hyperstable adaptive recursive filter (HARF) algorithm which represents the first technique proposed for adaptive IIR filtering was developed. The convergence property of HARF is provable. The SHARF algorithm was developed based on HARF, and most of HARF desirable properties were preserved while reducing the required computation (C.F.N. Cowan et 1985).

On the other hand, Volterra series filters and Radial Bias Function (RBF) networks may be a choice to solve this problem. Although the nonlinear FIR concepts with Volterra series filters can be utilized directly simply by extending the regression vector to include polynomial input terms, but the number of filter parameters required may become very large. 
In recent years, radial basis neural network architectures are introduced for the noise cancellation problem. It is shown that interpreting the noise and vibration cancellation concept in terms of a system identification problem provides a framework for the design of neural network architectures that provide a solution to this problem (Steve A. Billings, 1993).

This paper will introduce the property of TbDyFe magnetostrictive actuator and computer control experiments system at first. Then, a simple hyperstable adaptive recursive filter algorithm based on nonlinear model is proposed. And by using the radial basis based neural networks to obtain the nonlinear model of magnetostrictive actuator is presented in section 3. At last, section 4 will be devoted to the active vibration control results of real-time experiments.

\section{MAGNETOSTRICTIVE ACTUATOR AND EXPERIMENT SYSTEM OF REAL-TIME CONTROL}

Using zone melting unidirectional solidification, the relationship of unidirectional solidification preferred orientation, microstructure and magnetostrictive property of TbDyFe alloys has investigated.

The investigation and application to active vibration control of actuators using TbDyFe alloys are developing. As a smart material, TbDyFe can convert the change of external pressure into change of the magnetic field, which can be determined by external coil, and alter rod shape while external magnetic fields changing. Thus, smart sensor actuator system can be built when the alloys controlled by external electrical signal.

But as well know, the TbDyFe actuator is a nonlinear plant with magnetic hysteresis loop, therefore its position and active vibration control is a complex problem.

With multiple frequency input, the output stroke (measured by displacement transducer) vs. input current characteristics of TbDyFe actuator is nonlinear and exhibit magnetic hysteresis loop, due to the material property of TbDyFe. Fig. 1 shows experimental results when the input signal is complex sine $(40 \mathrm{~Hz}, 60 \mathrm{~Hz}, 80 \mathrm{~Hz})$.

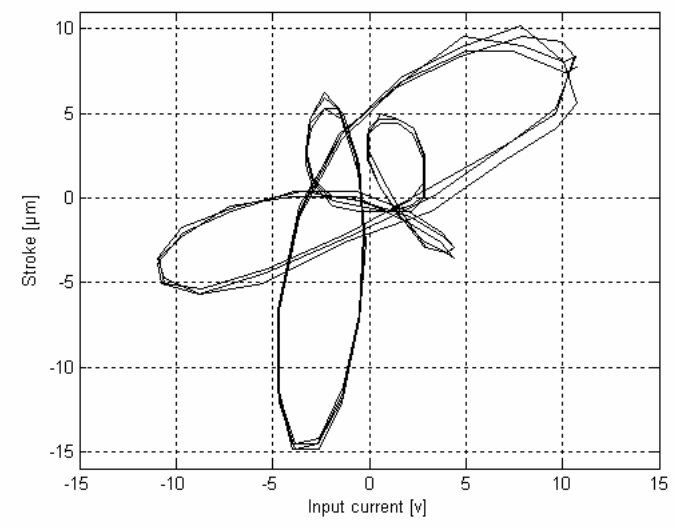

Figure 1. Output stroke vs. applied current (complex sine of $40 \mathrm{~Hz}, 60 \mathrm{~Hz}$ and $80 \mathrm{~Hz}$ ) of TbDyFe actuator

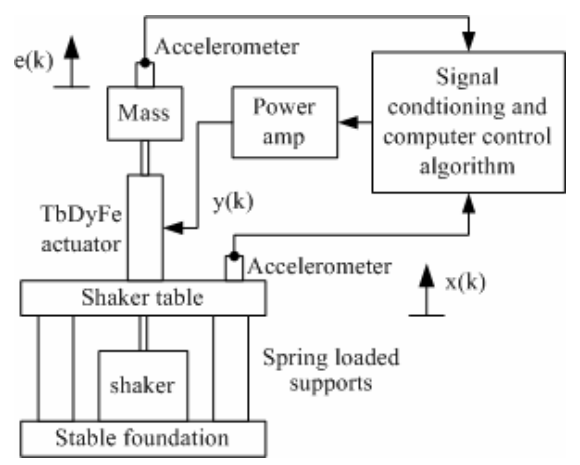

Figure 2. Control loop connection and computer control system with TbDyFe actuator

One degree of freedom magnetostrictive vibration control system was constructed, which is shown in Figure 2.

The piezoelectric accelerometer and additional signal conditioning equipments used to sense the error signals. Acceleration $e(k)$ on mass is measured by an accelerometer $\left(17.5 \mathrm{pc} / \mathrm{ms}^{-2}\right)$ to produce voltages $e(k)$ which is low-pass filtered $(200 \mathrm{~Hz})$, amplified (gain 1000), sampled by analog to digital converters $(1000 \mathrm{~Hz}$ sampling frequency), and then operated on by a industrial control computer (IPC610P) which executed a control algorithm based on the simple hyperstable adaptive recursive filter algorithm.

\section{SHARF ALGORITHM BASED ON NONLINEAR MODEL AND RADIAL BASIS NEURAL NETWORK}

\subsection{SHARF algorithm based on nonlinear model}

A Simple Hyperstable Adaptive Recursive Filter algorithm was developed and applied to the active vibration control. 


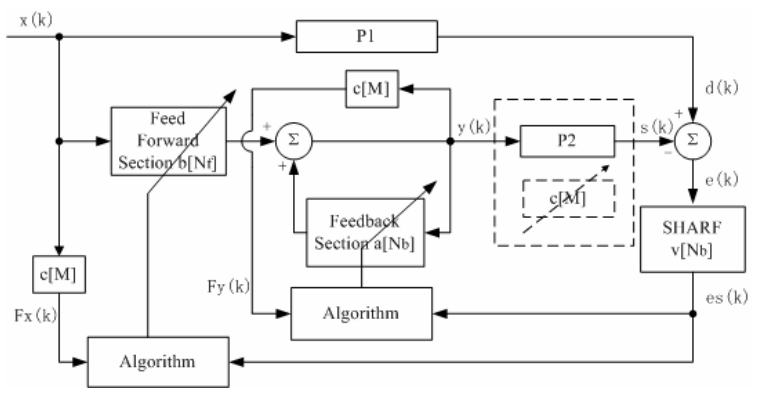

Figure 3. Implementation of Simple Hyperstable Adaptive Recursive Filter (SHARF)

The SHARF implementation is shown in Figure 3 for use in the following discussion.

The symbol $x(k)$ represents the vibration disturbance generated by shaker, P1 denotes the transfer function of the primary channel of the system to generate uncontrollable undesirable vibrational component $d(k)$ at the output sensor location, $p 2$ is the transfer function of the second channel whose response to the active control signal $y(k)$ is represented by $s(k)$. The output vibration disturbance $e(k)$ is picked up by output sensor and sent to two adaptive filter with input sensor signal $x(k)$ for active control signal generation.

The error signal at instant $\mathrm{k}$ is the sum of outputs from both primary and secondary channel which can expressed as:

$$
\begin{gathered}
e(k)=P 1(z) x(k)+P 2(z) y(k) \\
y(k)=\sum_{i=0}^{N_{f}} b_{i}(k) x(k-i)+\sum_{j=0}^{N_{b}} a_{j}(k) y(k-i)
\end{gathered}
$$

Where, $A(k)=\left[a_{1}(k) a_{2}(k) \cdots a_{N_{b}}(k)\right]^{T} \in R^{N_{b}}$

$$
B(k)=\left[b_{1}(k) b_{2}(k) \cdots b_{N_{f}}(k)\right]^{T} \in R^{N_{f}}
$$

and $a_{i}(k) \in R, i=1,2, \cdots, N_{b}$ are weight vectors of feed $b_{j}(k) \in R, j=1,2, \cdots, N_{f}$

feedback section and forward section at time $k$.

The SHARF algorithm based on the nonlinear model is expressed as following (Treichler 1978):

$$
\begin{aligned}
& a_{j}(k+1)=a_{j}(k)-2 \mu e_{s}(k) f_{y}(k-j) \\
& b_{j}(k+1)=b_{j}(k)-2 \mu e_{s}(k) f_{x}(k-j) \\
& i=1,2, \cdots, N_{f} ; j=1,2, \cdots, N_{b}
\end{aligned}
$$

where the auxiliary process $f_{x}(k)$ and $f_{y}(k)$ :

$$
f_{x}(k)=\sum_{l=0}^{M} c_{l} x(k-l), f_{y}(k)=\sum_{l=0}^{M} c_{l} y(k-l)
$$

To set SHARF moving-average parameter $v_{i}=-a_{i}$, so the moving-average process $e_{s}(k)$ is:

$$
e_{s}(k)=e(k)+\sum_{i=1}^{N_{b}}\left(-a_{i}\right) e(k-i)
$$

$\mu$ is iteration step. It is proved that when weight vectors $A$ and $B$ in (3.3) are adjusted according to output error $e(k)$, the error $e(k)$ will approach zero. As mentioned, that in (3.3) $f x(k)$ and $f y(k)$ is the linear function which is obtained to approximate the plant model $P 2$ In Figure 3. However since the magnetostrictive actuator is a complex nonlinear plant with magnetic hysteresis loop, the SHARF algorithm based on linear model is no longer suitable. If we could obtain the approximate nonlinear model of magnetostrictive actuator under given multiple frequency input as shown in Figure 1, then the SHARF algorithm based on nonlinear model could be express as the same equation as (3.3), but where:

$$
\begin{aligned}
& f_{x}(k)=f_{1}(X(k)) \\
& f_{y}(k)=f_{2}(Y(k))
\end{aligned}
$$

They are nonlinear functions, which approximates the nonlinear plant model, i.e., the magnetostrictive actuator model under given multiple frequency input.

\subsection{Radial basis neural network to approximate the nonlinear model}

Now the problem is how to obtain the nonlinear model of magnetostrictive actuator under given multiple frequency input. To solve this problem, the radial basis neural network is used to set up off-line modeling procedure.

Figure 4 shows the radial basis neural network. The network is three layers networks, in which the first layer connect to the second layer directly without any weighting process and the output layer is linear combination. Nonlinear mapping of input vector will be expressed by hide layer. In Figure 4, reference vectors

$$
r_{i}(k) \in R^{M}, i=0,1, \cdots, M-1
$$

and weight vector $W(k) \in R^{M}$ can be identified by an independent offline process (P.S. R. Diniz, 2002): $w_{i}(k+1)=w_{i}(k)+2 \mu_{w} e(k) f\left[d_{i}(k)\right]$

$$
r_{i}(k+1)=r_{i}(k)+2 \mu_{r} e(k) f\left[d_{i}(k)\right] w_{i}(k) \frac{X(k)-r_{i}(i)}{\sigma_{i}^{2}}
$$

$i=1,2, \cdots, M-1$

Where, $X(k)$ is input vector, $\mu_{w}$ and $\mu_{r}$ is iteration step, $\sigma_{i}$ is radius parameter, $d_{i}(k)=\left\|X(k)-r_{i}(k)\right\|$ is

Euclidean distance between input vector and reference vectors, and $f(\cdot)$ is nonlinear function:

$$
f\left(d_{i}(k)\right)=\exp ^{\frac{-d_{i}^{2}(k)}{\sigma_{i}^{2}(k)}}
$$

Thus, the auxiliary process $f_{x}(k), f_{y}(k)$ in (3.6) can be expressed as:

$$
\begin{aligned}
& f_{x}(k)=\sum_{i=0}^{M} w_{i}(k) f\left[\left\|X(k)-r_{i}(k)\right\|\right] \\
& f_{y}(k)=\sum_{i=0}^{M} w_{i}(k) f\left[\left\|Y(k)-r_{i}(k)\right\|\right]
\end{aligned}
$$




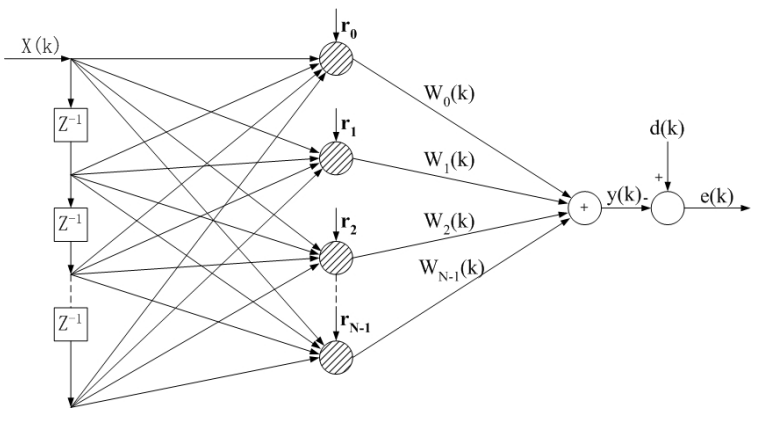

Figure 4. Radial basis neural network

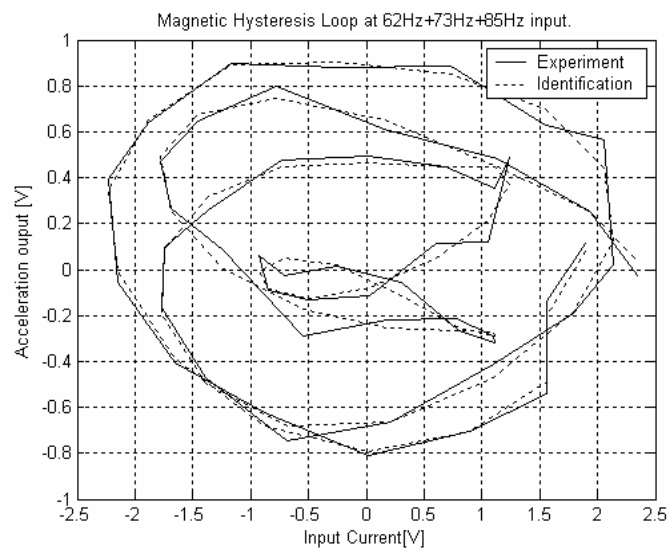

Figure 5. Magnetic hysteresis loop of nonlinear model (dash dot line) and experiment (solid line)

\section{EXPERIMENTAL RESULTS}

From $5.0 \mathrm{~Hz}$ to $100.0 \mathrm{~Hz}$, the resonant frequencies $60.2 \mathrm{~Hz}$ and $73.9 \mathrm{~Hz}$ of passive vibration control platform were founded. The first mode is most important because the vibration amplitude caused by vibration of $60.2 \mathrm{~Hz}$ is larger than any other frequencies in the area.

The tap length $N_{b}$ is 21 , tap length $N_{f}$ is $20, M$ is 10 , iteration step $\mu$ is $0.001, \mu_{w}$ is $0.1, \mu_{r}$ is 0.9 , other parameters are given in table 1.

Figure 5 shows the nonlinear model output (solid line is experimental magnetic hysteresis loop and dash dot line is identification one) in complex sine input $(60 \mathrm{~Hz}, 73 \mathrm{~Hz}$ and $85 \mathrm{~Hz})$. Notice that instead of the stroke regarded as the output of actuator in Figure 1, in Figure 5 the measured acceleration at the top of actuator is regarded as the output. Since the curve is complex, here only a part of the curve is shown by Figure 5. All the parameters about the nonlinear model are given in table 2 and table 3 .

Figure 6 and Figure 7 shows experimental curves of one freedom vibration control when the platform excited by the complex sine input.

In $1000 \mathrm{~Hz}$ sample frequency, acceleration measure- ments $e(k)$ from up accelerometers mounted on mass, and computer output control signal $y(k)$ are plotted in Figure 6. From 1 to 1000 of total 5000 samples, the mass is excited only by shaker, in which peak-to-peak value is about 1.18 volts. When the active control is turned on from 1001 samples, vibration acceleration reduced within 230 samples (about 0.23 seconds). From 4000 samples, the measured peak-to-peak value was reduced to 0.015 volts. An attenuation of about $30 \mathrm{~dB}$ is achieved. Figure 7 shows the comparison in frequency domain the acceleration measured from up accelerometer before and after the active control turned on.

\section{Table 1 Parameters of SHARF: $a$ and $b$}

\begin{tabular}{cccccc}
\hline $\mathrm{i}$ & \multicolumn{5}{c}{$\mathrm{a}_{\mathrm{i}}$ and $\mathrm{b}_{\mathrm{i}}$} \\
\hline \multicolumn{5}{c}{$\mathrm{a}_{\mathrm{i}}$} \\
$\mathrm{i}=1 \sim 5$ & 0.0241 & 0.0157 & 0.0030 & -0.0122 & -0.0280 \\
$\mathrm{i}=6 \sim 10$ & -0.0424 & -0.0533 & -0.0587 & -0.0569 & -0.0471 \\
$\mathrm{i}=11 \sim 15$ & -0.0298 & -0.0071 & 0.0178 & 0.0406 & 0.0569 \\
$\mathrm{i}=16 \sim 20$ & 0.0628 & 0.0566 & 0.0387 & 0.0124 & -0.0180 \\
$\mathrm{i}=21$ & \multicolumn{5}{c}{-0.0474} \\
$\mathrm{c}$ \\
$\mathrm{i}=1 \sim 5$ & -0.0006 & -0.0099 & -0.0160 & -0.0204 & -0.0243 \\
$\mathrm{i}=6 \sim 10$ & -0.0285 & -0.0328 & -0.0370 & -0.0401 & -0.0402 \\
$\mathrm{i}=11 \sim 15$ & -0.0354 & -0.0241 & -0.0065 & 0.0157 & 0.0395 \\
$\mathrm{i}=16 \sim 20$ & 0.0605 & 0.0741 & 0.0761 & 0.0640 & 0.0378 \\
\hline \multicolumn{5}{c}{}
\end{tabular}

Table 2 Parameters of nonlinear model: $\mathrm{W}$ and $\sigma$

\begin{tabular}{cccccc}
\hline $\mathrm{i}$ & \multicolumn{5}{c}{$\mathrm{W}_{\mathrm{i}}$ and $\sigma_{i}$} \\
\hline \multicolumn{5}{c}{$\mathrm{W}_{\mathrm{i}}$} \\
$\mathrm{i}=1 \sim 5$ & 2.3790 & -0.6062 & 0.0498 & 0.0563 & -0.8579 \\
$\mathrm{i}=6 \sim 10$ & 0.2487 & -2.1296 & -1.6600 & -1.0227 & 0.1350 \\
\multicolumn{5}{c}{$\sigma_{i}$} \\
$\mathrm{i}=1 \sim 5$ & 8.1917 & -0.2422 & 0.3724 & 0.3718 & 3.8773 \\
$\mathrm{i}=6 \sim 10$ & 0.2908 & 8.5718 & 0.0643 & -0.0548 & 0.3442 \\
\hline
\end{tabular}

Table 3 Parameters of nonlinear model: $\boldsymbol{r}_{\mathrm{ij}}$

\begin{tabular}{cccccc}
\hline $\mathrm{i}$ & \multicolumn{5}{c}{$\mathrm{r}_{\mathrm{i}, \mathrm{j}}$} \\
\hline $\mathrm{i}=1, \mathrm{j}=1 \sim 5$ & -1.3725 & -1.7378 & -1.8922 & -1.7930 & -1.4645 \\
$\mathrm{i}=1, \mathrm{j}=6 \sim 10$ & -1.0974 & -0.8365 & -0.8494 & -1.1642 & -1.7087 \\
$\mathrm{i}=2, \mathrm{j}=1 \sim 5$ & -0.5486 & -0.1807 & 0.0654 & 0.1997 & 0.0583 \\
$\mathrm{i}=2, \mathrm{j}=6 \sim 10$ & -0.0998 & -0.2990 & -0.3512 & -0.2032 & 0.2024 \\
$\mathrm{i}=3, \mathrm{j}=1 \sim 5$ & -0.0120 & 0.0266 & 0.0448 & 0.0481 & 0.0314 \\
$\mathrm{i}=3, \mathrm{j}=6 \sim 10$ & 0.0118 & -0.0005 & -0.0014 & 0.0081 & 0.0370 \\
$\mathrm{i}=4, \mathrm{j}=1 \sim 5$ & -0.0022 & 0.0331 & 0.0480 & 0.0480 & 0.0323 \\
$\mathrm{i}=4, \mathrm{j}=6 \sim 10$ & 0.0130 & 0.0042 & 0.0052 & 0.0143 & 0.0407 \\
$\mathrm{i}=5, \mathrm{j}=1 \sim 5$ & 0.8908 & 0.7072 & 0.3096 & -0.2083 & -0.6751 \\
$\mathrm{i}=5, \mathrm{j}=6 \sim 10$ & -0.9125 & -0.7505 & -0.0425 & 1.1614 & 2.6041 \\
$\mathrm{i}=6, \mathrm{j}=1 \sim 5$ & -0.1943 & -0.0390 & 0.0698 & 0.0561 & 0.0220 \\
$\mathrm{i}=6, \mathrm{j}=6 \sim 10$ & -0.0870 & -0.1478 & -0.1555 & -0.0528 & 0.1461 \\
$\mathrm{i}=7, \mathrm{j}=1 \sim 5$ & 1.4184 & 2.0554 & 2.4020 & 2.3950 & 2.1022 \\
$\mathrm{i}=7, \mathrm{j}=6 \sim 10$ & 1.6673 & 1.2995 & 1.1562 & 1.2331 & 1.4307 \\
$\mathrm{i}=8, \mathrm{j}=1 \sim 5$ & 0.0136 & 0.0263 & 0.0089 & -0.0145 & -0.0414 \\
$\mathrm{i}=8, \mathrm{j}=6 \sim 10$ & -0.0500 & -0.0313 & 0.0197 & 0.0923 & 0.1722 \\
$\mathrm{i}=9, \mathrm{j}=1 \sim 5$ & -0.0472 & 0.0054 & 0.0190 & 0.0106 & -0.0285 \\
$\mathrm{i}=9, \mathrm{j}=6 \sim 10$ & -0.0564 & -0.0638 & -0.0267 & 0.0531 & 0.1682 \\
$\mathrm{i}=10, \mathrm{j}=1 \sim 5$ & -0.0199 & 0.0432 & 0.0735 & 0.0493 & 0.0359 \\
$\mathrm{i}=10, \mathrm{j}=6 \sim 10$ & -0.0177 & -0.0174 & -0.0053 & 0.0398 & 0.1276 \\
\hline & & & & & \\
\hline
\end{tabular}



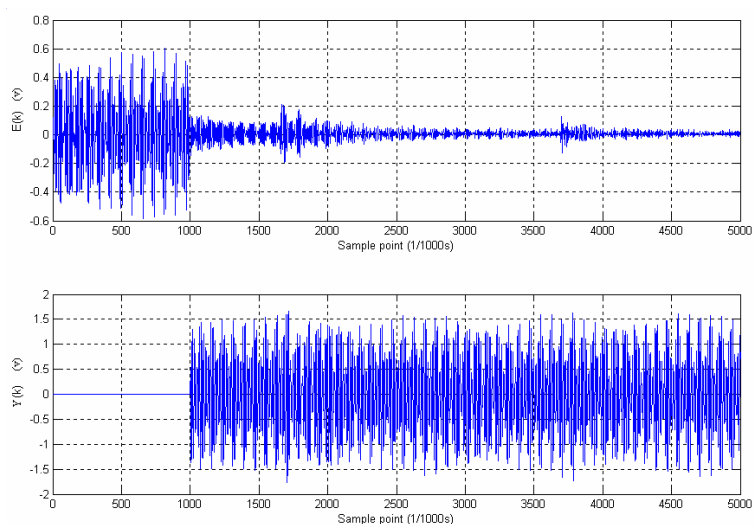

Figure 6. Experimental curves of vibration control: The evolution of the error signal $e(k)$ and output control signal $y(k)$

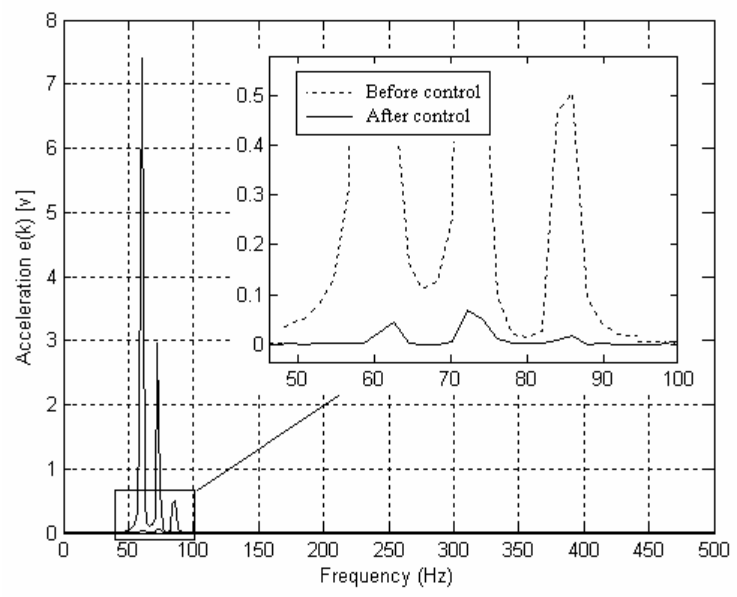

Figure 7. Experimental curves of vibration control: Comparison of frequency domain response of active vibration control

\section{CONCLUSION}

This paper proposed a Simple Hyperstable Adaptive Recursive Filter (SHARF) algorithm based on the nonlinear model which is obtained by using radial basis neural network. The experimental result show that the proposed approach is real-time efficient. An attenuation of about $30 \mathrm{~dB}$ is achieved. Notice that since the magnetic hysteresis loop is a complex nonlinear function, further research is focus on some problems like the global convergence etc.

\section{REFERENCES}

C.F.N. Cowan, P.M. Grant. (1985), Adaptive Filters (Prentice-Hall series in signal processing), Prentice-Hall, Inc., New Jersey.

Michael D. Bryant, Benito Fernandez, Ning Wang. (1993), Active vibration control in structures using magnetostrictive Terfenol with feedback and/or neural network controllers, Journal of Intelligent Material Systems and Structures, Vol. 4 Oct.

Paulo S.R. Diniz. (2002), Adaptive Filtering: Algorithms and Practical Implementation, Kluwer Academic Publishers, 2nd edition.

Steve A. Billings and Chi F. Fung. (1995) Recurrent Radial Basis Function Networks for Adaptive Noise Cancellation, Neural Networks, Vol.8, No.2, PP. 273-290.

Treichler, J. R., Larimore, M. G., and Johnston, C. R. (1978), Simple Adaptive IIR Filtering, Proceedings IEEE International Conference Acoustics, Speech, and Signal Processing (ICASSP), pp. 118-122.

X.Tan, R.Venkataraman, and P.S.Krishnaprasad. (2001), Control of hyseresis: Theory and experimental results, Modeling, Signal Processing, and Control in Smart Structures, vol. 4326 of SPIE, pp. 101-112.

Z. Jason Geng. (1995), An intelligent control system for multiple Degree-Of-Freedom vibration isolation, Journal of Intelligent Material Systems and Structures, Vol. 6 Nov.

Z. Jason Geng and Leonard S. Haynes. (1994), Six Degree-of-Freedom Active Vibration Control Using the Stewart Platforms, IEEE Transactions on control systems technology, 2(1), pp.45-53. 